\title{
PRODUCCTIVIDAD DE BIOMASA FORRAJERA AÉREA DE VARIEDADES DE TRIGO (Triticum aestivum L.) Y SU RELACIÓN CON COORDENADAS CROMÁTICAS DERIVADAS DE CAMARA DIGITAL
}

\section{RELATING THE AERIAL FORAGE BIOMASS PRODUCTIVITY OF WHEAT VARIETIES (Triticum aestivum L.) TO DIGITAL CAMERA-DERIVED CHROMATIC COORDINATES}

\author{
José Luis Tiedemann ${ }^{1}$
}

\begin{abstract}
Resumen
La productividad de biomasa forrajera aérea (PBFA) de variedades de trigo (Triticum aestivum L.) fue cuantificada y comparada mediante un ANAVA en un establecimiento de la Colonia El Simbolar, Santiago del Estero, Argentina. La PBFA de las variedades fue relacionada con coordenadas cromáticas derivadas de cámara digital y a partir de ellas fueron ajustados modelos lineales. No se encontraron diferencias significativas en la PBFA acumulada entre variedades, la misma osciló entre $4.4 \mathrm{tn} \mathrm{ms} \mathrm{ha}^{-1}$ y $5.6 \mathrm{tn} \mathrm{ms} \mathrm{ha}^{-1}$. Se encontraron diferencias significativas en la PBFA en el tercer $\left(\mathrm{R}^{2}=0.55 ; p<0.01\right)$ y cuarto corte $\left(\mathrm{R}^{2}=0.47 ; p<0.01\right)$, siendo respectivamente las variedades Yarará (2.1 tn $\left.\mathrm{ms} \mathrm{ha}^{-1}\right)$ y Gladiador $\left(1.1 \mathrm{tn} \mathrm{ms} \mathrm{ha}^{-1}\right)$ las de mayor productividad. Se encontraron fuertes relaciones inversas $(r-0.99 ; r-0.88)$ y directas $(r$ 0.96) entre la PBFA de variedades y la coordenada cromática integrada $\Sigma$ cct. Se destaca que la simple coordenada cromática $\Sigma$ ccr (red) tuvo una fuerte relación inversa con la PBFA de las variedades Yarará y Gladiador, con un $r-0.99$, para ambas variedades. Los modelos lineales ajustados entre la PBFA y coordenadas cromáticas presentaron un elevado poder explicativo con un $\mathrm{R}^{2}$ aj que osciló entre 0.71 y 0.99 . Las coordenadas cromáticas derivadas de cámaras digitales posibilitan, de manera simple y económica, cuantificar la productividad de biomasa forrajera aérea de variedades de trigo.
\end{abstract}

Palabras clave: RGB, sensores cercanos, modelos lineales, cromaticidad.

\begin{abstract}
The aerial forage biomass productivity (AFBP) of wheat varieties (Triticum aestivum L.) was quantified and compared using ANAVA in a facility located at Colonia El Simbolar, Santiago del Estero, Argentina. AFBP of the varieties were related to digital camera-derived chromatic coordinates and from the data lineal models were adjusted. Significant differences in the accumulated AFBP (which ranged from 4.4 and $5.6 \mathrm{tn} \mathrm{ms}$ ha-1) among varieties were not found. On the contrary, significant differences were found in the AFBP of the third $\left(\mathrm{R}^{2}=0.55 ; \mathrm{p}<0.01\right)$ and fourth $\left(\mathrm{R}^{2}=0.47 ; \mathrm{p}<0.01\right)$ cut, being Yarara $\left(2.1 \mathrm{tn} \mathrm{ms} \mathrm{ha}{ }^{-1}\right)$ and Gladiator $\left(1.1 \mathrm{tn} \mathrm{ms} \mathrm{ha-}{ }^{1}\right)$ varieties those with the highest productivity: Strong inverse $(r-0.99 ; r-0.88)$ and straight $(r$ 0.96) relationships were found among the AFBP of the varieties and the integrate chromatic coordinate $\Sigma$ cct. It is highlighted that the simple chromatic coordinate $\Sigma$ cet (red) showed strong inverse relationship with the AFPB of the varieties Yarara and Gladiator with a $r-0.99$ for both varieties. The lineal models adjusted by the AFPB and the chromatic coordinate presented a high explanatory power with a $\mathrm{R}^{2} \mathrm{Aj}$ ranging between 0.71 and 0.99 . The digital camera-derived chromatic coordinates allow for the aerial forage biomass productivity of wheat varieties to be quantified easily and economically.
\end{abstract} Key words: RGB, near sensor, lineal models, chromaticity.

\section{Introducción.}

La productividad primaria neta terrestre (PPN), es la integración temporal del incremento positivo de biomasa de la vegetación terrestre (Field et al., 1995; Fensholt, 2003; Fensholt et al., 2006). Es el nuevo carbono fijado como biomasa en tallos, hojas y raíces de todas las plantas en el ecosistema (Fensholt, 2003; Fensholt et al., 2006). Los Índice de Vegetación fueron ampliamente utilizados para determinar parámetros como: el índice de área foliar (Tucker \& Sellers, 1986), la radiación fotosintéticamente activa absorbida (Asrar et al., 1984; Dye \& Goward, 1993; Nouvellon et al., 2000), la tasa fotosintética y concentración de clorofila (Tucker, 1979) y la productividad primaria neta global (Field et al., 1995) entre otros. Al respecto, fueron desarrollados una gran cantidad de trabajos, como el de 
Field et al. (1995) quienes propusieron un simple modelo de PPN global, basado en la amplia relación existente entre los principios ecológicos, los recursos climáticos, las características de las especies y la PPN. A su vez, Goetz et al. (1999) mediante un modelo de eficiencia de productividad conducido a partir de observaciones satelitales, generaron mapas de productividad primaria bruta y neta, respiración autótrofa, biomasa y otras variables biofísicas del bosque Boreal de Canadá.

A nivel regional, mediante series temporales del NDVI MODIS, Tiedemann (2015) determinó la productividad primaria neta aérea (PPNA) de sistemas pastoriles de Panicum maximun en el Dpto. Moreno, Santiago del Estero. Mediante series temporales NDVI VGT, Tiedemann (2011a) y Tiedemann (2011b) determinó, respectivamente, la dinámica espacial y temporal del NDVI de las coberturas vegetales y los parámetros fenológicos de las subregiones naturales Chaco Semiárido y Chaco Serrano de la Provincia de Santiago del Estero, Argentina.

A escala predial, Aase \& Siddoway (1981) utilizaron el RATIO y el NDVI derivados de MSS Landsat para determinar la estación de crecimiento de cultivares de trigo y relacionarlos con la producción total de materia seca. Ellos determinaron fuertes relaciones entre la producción de materia seca total con el RATIO $\left(\mathrm{R}^{2}=0.89\right)$ y el NDVI $\left(\mathrm{R}^{2}=0.94\right)$. Entre los trabajos precursores desarrollados a partir de cámaras digitales podemos mencionar los de Wiegand et al. (1979) y Aase \& Siddoway. (1981) quienes encontraron fuertes relaciones entre el índice de área foliar (IAF) y la materia seca de hojas de trigo con índices de vegetación. Lukina et al. (1999) evaluaron técnicas de procesamiento de imágenes digitales y estimaron porcentaje de cobertura y biomasa en trigo; ellos determinaron fuertes relaciones entre el porcentaje de cobertura y el NDVI, concluyendo que los datos derivados de cámaras digitales poseen un buen valor predictivo. Adamsen et al. (1999) cuantificaron la tasa de senescencia o variación del verde en trigo, mediante la relación Green/Red derivado de una cámara digital y contrastaron los resultados con el NDVI derivado de un espectrómetro manual y con la concentración de clorofila en hoja derivada del SPAD 502 meter, concluyendo que las cámaras digitales tienen gran potencialidad para ser utilizadas como herramientas de monitoreo del estado de los cultivos. Kawashima \& Nakatani (1998) desarrollaron un algoritmo para determinar el contenido de clorofila de las hojas mediante una cámara de video portátil y una computadora personal.

El avance en el desarrollo de las cámaras digitales RGB estándar (Ahrends et al., 2008) impulsó, de manera simultánea, el desarrollo de software para procesamiento y análisis de imágenes digitales (Ahrends et al., 2008; Mizunuma et al., 2011; Sakamoto et al., 2012). Las cámaras digitales actuales pueden considerarse sensores cercanos de teledetección de alta resolución espacial y temporal, poseen un sistema cartesiano de captura de colores en el espacio rojo $(\mathrm{R})$, verde $(\mathrm{G})$ y azul $(\mathrm{B})$, donde los números digitales RGB (DN) posibilitan registrar eventos de la vegetación (Gitelson et al., 2002; Sonnetag et al., 2011; Sakamoto et al., 2012). De acuerdo a Gitelson et al. (2002), las intensidades de las reflectancia verde y roja son eficientes alternativas para determinar propiedades biofísicas en cultivos agrícolas como el trigo (Triticum aestivum L.). En este sentido, Ahrends et al. (2009) relacionaron la coordenada cromática green $(g)$ derivado de cámara digital con la productividad primaria bruta a escala de ecosistemas forestales. Las mejores relaciones fueron encontradas en la primavera, durante los picos de verde de la vegetación, estas oscilando entre $\mathrm{R}^{2} 0.86$ y $\mathrm{R}^{2}$ 0.93. A su vez, Ide \& Oguma (2010) desarrollaron índices de corrección atmosférica, o filtros climáticos utilizando fracciones del visible e infrarrojo cercano derivados de imágenes derivadas de cámaras digitales para el monitoreo de cultivos. Baret et al. (2010) evaluaron la eficiencia de imágenes digitales tomadas en sentido perpendicular a la línea de siembra de trigo en un ángulo de $57.5^{\circ}$ para determinar el índice de área verde (IAV), demostrando que es un excelente estimador del IAF en estado vegetativo de trigo. A su vez, Woebbecke et al. (1995) lograron la mejor clasificación entre las plantas verdes y su ambiente mediante el índice de color $2 g-r-b$ y la coordenada cromática verde $(g)$.

Las cámaras digitales, de alta resolución espacial y temporal, son comercialmente accesibles y disponibles, y son una poderosa herramienta para el monitoreo de vegetación agrícola y natural (Sakamoto et al., 2011).

Con base a lo antes expuesto los objetivos de este trabajo fueron: a) determinar la productividad de biomasa forrajera aérea (PBFA) de variedades de trigos Klein (Triticum aestivum L.); b) relacionar la productividad de biomasa forrajera seca aérea por corte y acumulada con coordenadas cromáticas; y c) generar modelos lineales de productividad de biomasa aérea por cultivar.

\section{Materiales y métodos.}

El ensayo fue realizado en el establecimiento cuyo nombre y/o razón social es "JB", ubicado en la Colonia El Simbolar, Dpto. Robles, Provincia de Santiago del Estero, Argentina, y cuyas coordenadas centrales son Lat $-27.73^{\circ}$ y $\log -63.89^{\circ}$ (Figura 1). La Colonia El Simbolar está situada en la Planicie fluvio-eólica chaqueña (Río Sali-Dulce), en los planos convexos y cauces antiguos del mega abanico aluvial distal (Angueira et al., 2007). El tipo de suelo de la zona del ensayo es Haplustol torriorténtico franco a franco limoso (Angueira et al., 2007). Según la clasificación climática de Köppen, el clima de la región es semiárido (BShw) (Torres Bruchmann, 1981). La precipitación es de $550 \mathrm{~mm}$, la evapotranspiración anual se ubica entre 

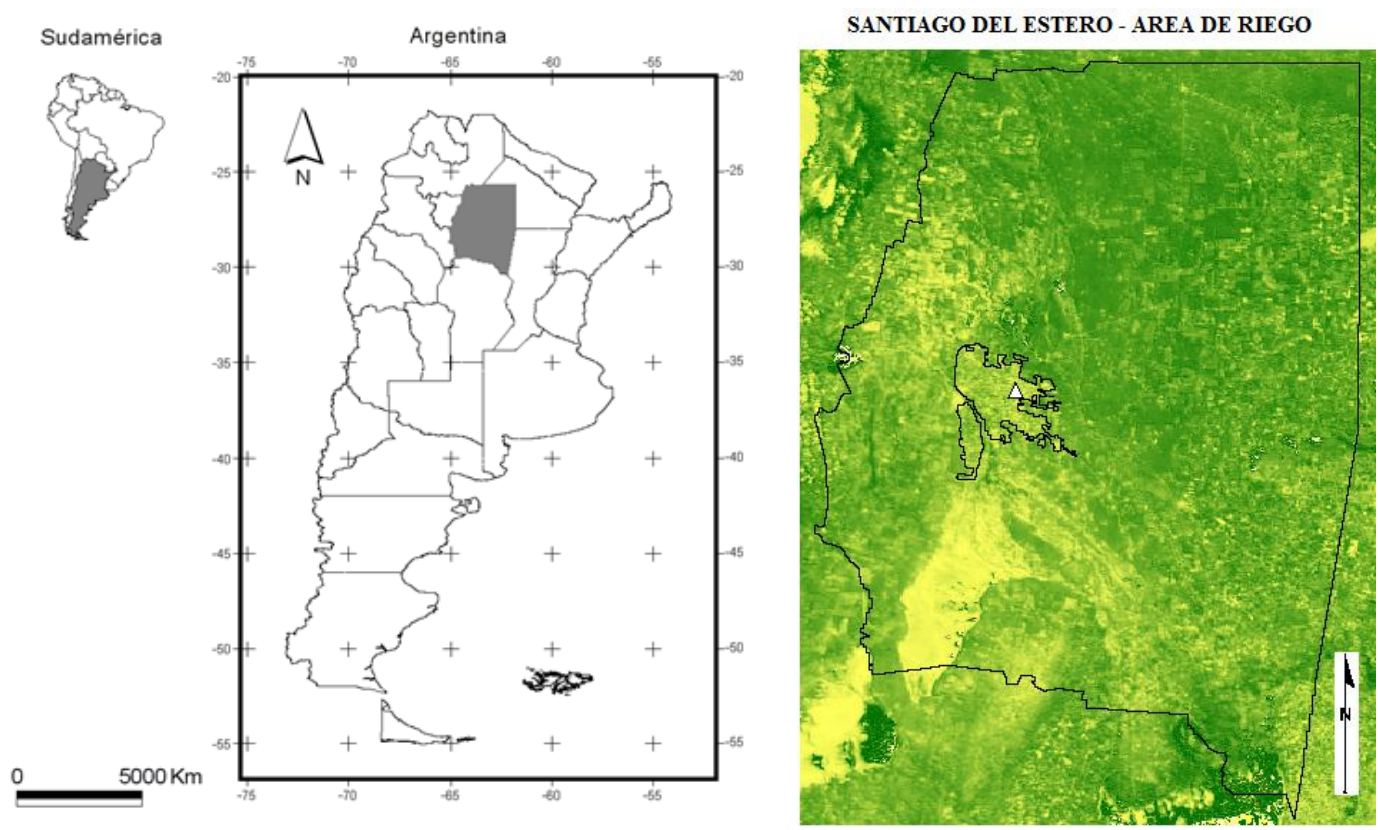

Figura 1. (Izquierda) Argentina en Sudamérica y Santiago del Estero en Argentina; (Derecha) área de riego del Río Dulce de Santiago del Estero y ubicación del establecimiento en el área de riego (triángulo blanco - coordenadas Lat $-27.73^{\circ}$; Log $-63.89^{\circ}$ ).

los 900-1 100 mm, con déficit hídrico todo el año, en un promedio de $550 \mathrm{~mm}$. La temperatura media anual histórica es de $27.7^{\circ} \mathrm{C}$. La temperatura media mensual del mes más caliente (enero) es de $28^{\circ} \mathrm{C}$ y la del mes más frio (julio) es de $16.3^{\circ} \mathrm{C}$ (Boletta, 2001).

El ensayo comprende a cuatro variedades comerciales del criadero Klein: un ciclo largo Gladiador y tres ciclo intermedio-largo Yarará, Serpiente y Titanio. Las características de las variedades, así como la información del criadero, se encuentran disponibles en http://trigoklein.com.ar (2017 ® Criadero Klein).

Las variedades fueron sembradas en un diseño completamente al azar, en parcelas de tres $\mathrm{m}^{2}$ de superficie con seis repeticiones por variedad. La fecha de siembra osciló entre el 29 de mayo al 01 de junio, la siembra fue en línea y con una densidad media efectiva de 234 semillas $\mathrm{m}^{-2}$. La biomasa forrajera aérea verde de cada variedad fue cortada en el centro de cada parcela utilizando un cuadrado de $1 \mathrm{~m}^{2}$, en estado vegetativo a una altura óptima de pastoreo bovino ( 30 $\mathrm{cm})$. El corte se realizó por encima de $\operatorname{los} 7 \mathrm{~cm}$, preservando vainas basales y área foliar remanente (Gardner, 1974). La materia verde fue recolectada y pesada $\left(\mathrm{kg} \mathrm{mv} \mathrm{m}^{-2}\right)$, de ella se extrajeron $3 \mathrm{sub}$ muestras al azar para determinar el contenido de humedad (\%

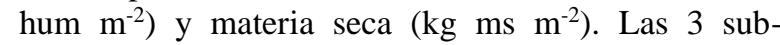
muestras fueron pesadas en verde, posteriormente secadas en horno microondas (Crespo et al., 2007) y pesadas nuevamente hasta peso constante, los valores obtenidos de materia seca de las tres sub muestras fueron promediados y llevado a tonelada (tn $\mathrm{ms} \mathrm{ha}^{-1}$ ). El porcentaje de humedad se obtuvo mediante la ecuación: \% de hum $=100 *[($ peso verde - peso seco $)$ / peso verde] (Gardner, 1974). Las parcelas fueron inundadas para la siembra y dos riegos post siembra $(\sim 150 \mathrm{~mm} /$ riego).

La productividad de biomasa forrajera aérea acumulada (PBFA) y por corte de las variedades, expresada en toneladas de materia seca por hectárea (tn $\mathrm{ms} \mathrm{m}^{-2}$ ), fue evaluada mediante un ANAVA en diseño completamente al azar (Balzarini et al., 2008). Se verificó normalidad con Shapiro-Wilks modificado y homogeneidad de varianza con la prueba de Levene (Balzarini et al., 2008). La comparación de medias se realizó mediante el test de comparaciones múltiples Tukey (Di Rienzo et al., 2008).

La PMSA acumulada (tn $\mathrm{ms} \mathrm{m}^{-2}$ ) y por corte fueron relacionadas con las coordenadas cromáticas integradas (Tabla 1) mediante el coeficiente de correlación de Pearson (r). El modelo de regresión lineal simple ( $\alpha$ 0.05 ), utilizado para la observación del tratamiento $i$ en la parcela $j$, fue $\mathrm{Y}_{\mathrm{ij}}=\mu+\tau_{\mathrm{i}}+\varepsilon$; donde $\mathrm{Y}_{\mathrm{ij}}$ son las observaciones del tratamiento $i$ en la parcela $j$; $\tau_{\mathrm{i}}$ es el efecto del tratamiento $i$ y $\varepsilon$ el término de error aleatorio asociado a la observación $\mathrm{Y}_{\mathrm{ij}}$. La variable dependiente (y) es la PBFA acumulada de cada variedad y la regresora $(x)$ las coordenadas cromáticas integradas. Los modelos lineales fueron evaluados en base al mejor ajuste del coeficiente de determinación ajustado $\left(\mathrm{R}_{\mathrm{Aj}}{ }\right)$ y mediante la observación de los gráficos de dispersión (residuos vs. predichos) (Balzarini et al., 2008). 


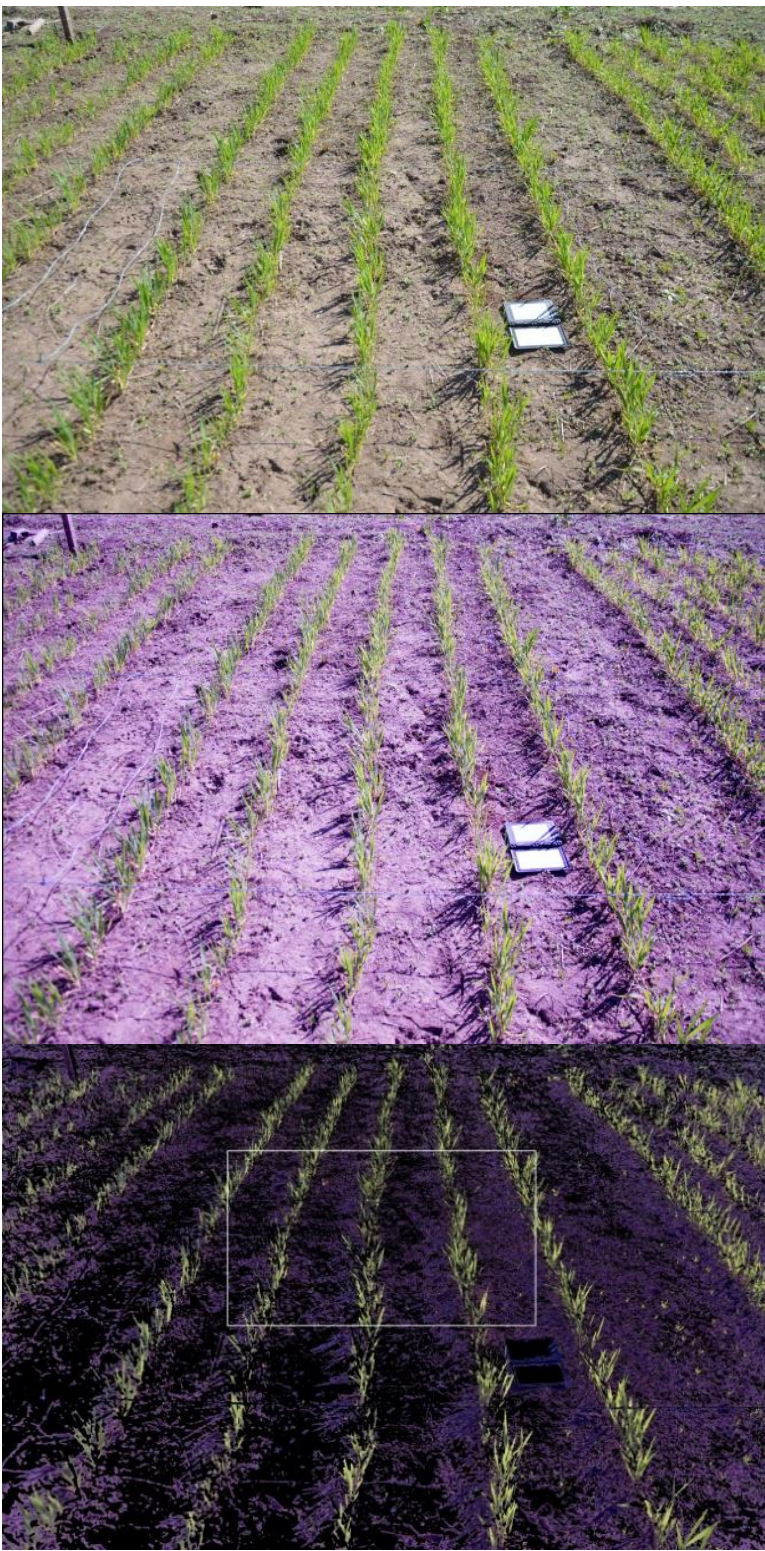

Figura 2. (Superior) imagen RGB de parcela de trigo; (centro) imagen RGB corregida mediante perfil DGN de variaciones de sensor y balance de blancos mediante la carta de reflectancia $18 \%$ Gray Balance; (Abajo) Imagen binaria y área de muestreo digital (polígono blanco).

Programación técnica de la cámara y fotografía.

Las imágenes fueron obtenidas con una cámara digital réflex Nikon D7100 (Nikon Corporation, Tokyo, Japón) con sensor de imagen CMOS de 23.5 x $15.6 \mathrm{~mm}, 24.1$ megapíxeles efectivos, lente AF-S

Tabla 1. Coordenadas cromáticas.

\begin{tabular}{ccc}
\hline $\begin{array}{c}\text { Coordinate chromatic total } \\
\text { (cct) }\end{array}$ & $\mathrm{r}+\mathrm{g}+\mathrm{b}$ & Saitoh et al. (2012) \\
$\begin{array}{c}\text { Simple coordinate chromatic } \\
(\mathrm{ccr})\end{array}$ & $\mathrm{r}$ & $\begin{array}{c}\text { Gillespie et al. } \\
(1986)\end{array}$ \\
\hline
\end{tabular}

NIKKOR 18-300 mm f/3.5-5.6G ED VR. El tamaño de fotograma seleccionado fue de $2.304 \times 1.536$ pixeles en formato RAW (Nikon Electronic Format) 14 bits de profundidad, 24 bit de resolución RGB (Ahrends et al, 2009). La cámara fue programada en modo automático y sensibilidad ISO 200 (Sakamoto et al., 2011).

La cámara fue configurada geométricamente en un trípode vertical con niveles de burbujas y graduación de ángulos, a $1 \mathrm{~m}$ de distancia de las parcelas y $1 \mathrm{~m}$ de altura del tope del follaje, el sensor fue orientado al centro de cada parcela con un ángulo de inclinación de 45 (Rasmussen et al., 2007) (Figura 2). Las imágenes fueron tomadas, a partir de los 15 días post siembra, una vez por semana, en días libres de nubes, al mediodía solar entre las $10.00 \mathrm{~h}$ y $14.00 \mathrm{~h}$ (Lee \& Lee, 2013; Inoue et al., 2015). El balance de blancos de las imágenes fue corregido tomando como imagen de referencia cromática la carta de reflectancia $18 \%$ Gray Balance $X$-Rite colocadas en área central de corte (Inoue et al., 2015) (Figura 2).

Procesamiento y digitalización de imágenes.

Para el calibrado del sensor de la cámara se generó un perfil en formato digital negativo (DGN - Adobe Systems Incorporate) a partir de una imagen de referencia cromática estándar Color Checker Passport (X-Rite, Incorporated). Las imágenes RAW (Nikon Electronic Format) fueron ajustadas linealmente en términos espectrales mediante el perfil de calibrado (DGN) con Adobe Cámera Raw (Adobe Systems Incorporate). Una vez calibradas se almacenaron en formato TIFF (Tagged Image File Format) estandarizadas a 8 bit (Min 0; Max 255). Los números digitales (ND) fueron extractados de cada parcela con un polígono digital de muestreo (Sonnetag et al., 2011) mediante el software ImageJ (Ferreira \& Rasband, 2016) (Figura 2). Las imágenes fueron clasificadas mediante el clasificador desarrollado por Yen et al. (1995), dando el valor de uno a la vegetación fotosintéticamente activa (Baret et al., 2010) y el valor cero al resto del background.

Coordenadas cromáticas.

Debido a que los ND extractados de las imágenes RGB son fuertemente influenciados por la iluminación de la escena, para suprimir el efecto se efectuó una transformación no lineal de RGB a coordenadas cromáticas (cc) r, g y b (Gillespie et al., 1986 y 1987; Woebbecke et al., 1995).

Las coordenadas cromáticas $(r, g, b)$ se derivan de la siguiente manera:

$r=\mathrm{R}^{*} / \mathrm{R}^{*}+\mathrm{G}^{*}+\mathrm{B}^{*} ; g=\mathrm{G}^{*} / \mathrm{R}^{*}+\mathrm{G}^{*}+\mathrm{B}^{*} ; \quad b=$ $\mathrm{B}^{*} / \mathrm{R}^{*}+\mathrm{G}^{*}+\mathrm{B}^{*}$ en donde los valores $\mathrm{R}^{*}, \mathrm{G}^{*} \mathrm{y} \mathrm{B}^{*}$ son los valores RGB normalizados (0-1) definidos como:

$\mathrm{R}^{*}=\mathrm{R} / \mathrm{Rm} ; \mathrm{G}^{*}=\mathrm{G} / \mathrm{Gm} ; \mathrm{B}^{*}=\mathrm{B} / \mathrm{Bm}$, siendo $\mathrm{R}, \mathrm{G}$ y $\mathrm{B}$ los números digitales de la vegetación fotosintéticamente activa del área de interés y $\mathrm{Rm}=$ $\mathrm{Gm}=\mathrm{Bm}=255$ (8 bit) (Gillespie et al., 1986; Woebbecke et al., 1995). Las coordenadas cromáticas 
Tabla 2. Comparación de medias de PBFA (tn $\mathrm{ms} \mathrm{ha}^{-1}$ ) por corte (fecha del corte) y acumulada entre variedades mediante test de Tukey.

\begin{tabular}{|c|c|c|c|c|c|}
\hline Variedad & $\begin{array}{c}\text { Corte 1 } \\
\text { 01.07.17 } \\
\text { tn } \mathrm{ms} \mathrm{ha}^{-1} \\
\end{array}$ & $\begin{array}{c}\text { Corte } 2 \\
\mathbf{2 0 . 0 7 . 1 7} \\
\text { tn } \mathrm{ms} \mathrm{ha}^{-1} \\
\end{array}$ & $\begin{array}{c}\text { Corte } 3 \\
\text { 12.08.17 } \\
\text { tn } \mathrm{ms} \mathrm{ha}^{-1}\end{array}$ & $\begin{array}{c}\text { Corte } 4 \\
\text { 03.09.17 } \\
\text { tn } \mathrm{ms} \mathrm{ha}^{-1} \\
\end{array}$ & $\begin{array}{l}\text { Acumulado } \\
\text { tn } \mathrm{ms} \mathrm{ha}^{-1}\end{array}$ \\
\hline SERPIENTE & $1.2 \mathrm{a}$ & $1.4 \mathrm{a}$ & $1.4 a$ & $0.5 \mathbf{a}$ & $5.0 \mathrm{a}$ \\
\hline TITANIO & $0.8 \mathrm{a}$ & $1.2 \mathrm{a}$ & $1.4 \mathrm{a}$ & 0.8ab & $4.5 \mathrm{a}$ \\
\hline GLADIADOR & $0.9 \mathrm{a}$ & $1.5 \mathrm{a}$ & $0.9 \mathrm{a}$ & $1.1 b$ & $4.4 \mathrm{a}$ \\
\hline YARARÁ & $1.2 \mathrm{a}$ & $1.2 \mathrm{a}$ & $2.1 \mathrm{~b}$ & $1.1 b$ & $5.6^{\mathrm{a}}$ \\
\hline
\end{tabular}

utilizadas en este trabajo se detallan en la Tabla 1. Las coordenadas cromáticas fueron temporalmente integradas ( $\left.\Sigma \mathrm{cc}_{\mathrm{ij}}\right)$ (Tucker, 1979), en donde $i$ es la cc de la semana $j$ hasta el momento del corte.

\section{Resultados.}

Se realizaron cuatro cortes en estado vegetativo durante todo el ciclo productivo de las variedades. El primero se realizó el 01.07.17, el segundo el 20.07.17, el tercero el 12.08.17 y el último el 03.09.17. No se encontraron diferencias significativas en la productividad primaria neta aérea acumulada entre variedades $\left(\mathrm{R}^{2}{ }_{\mathrm{Aj}}=0.24 ; p>0.05\right)($ Tabla 2$)$ pero sí en la productividad de las variedades en el tercer $\left(\mathrm{R}^{2}{ }_{\mathrm{Aj}}=\right.$ $0.55 ; p<0.01)$ y cuarto corte $\left(\mathrm{R}^{2}{ }_{\mathrm{Aj}}=0.47 ; p<0.01\right)$ (Tabla 2).

El $66.6 \%$ de los días que duró el ensayo tuvieron condiciones climáticas: nublado, parcialmente nublado y lluvia (www.smn.gob.ar), afectando las condiciones para la toma de registros fotográficos. En total se realizaron 5 registros fotográficos por parcela, dando un total de 120 imágenes RGB (RAW). Todas las imágenes fueron corregidas linealmente de variaciones de sensor, balance de blancos y transformadas a formato TIFF 8 bit. A partir de estas fueron confeccionadas las coordenadas cromáticas y posteriormente integradas ( $\Sigma$ cct y $\Sigma$ ccr).

En la Tabla 3 se presentan las relaciones $(r)$ entre coordenadas cromáticas y la PBFA por variedad. No se encontraron evidencias para rechazar las hipótesis de normalidad y homogeneidad de varianzas ( $\mathrm{p}>0.05$ ).

En la Tabla 4 se presentan los modelos lineales de mayor poder explicativo en base al coeficiente de determinación ajustado y gráficos de residuos vs. predichos con patrones aleatorios.

\section{Discusión.}

La productividad de biomasa forrajera aérea acumulada de los cultivares de trigo osciló entre las 4.4 y $5.6 \mathrm{tn} \mathrm{ms} \mathrm{ha}^{-1}$. De acuerdo a estos rendimientos podemos aseverar que los cultivares evaluados pueden ser utilizados como verdeos de invierno en establecimientos agro-ganaderos de la zona. Sin embargo, y de acuerdo al rendimiento en tn $\mathrm{ms} \mathrm{ha}^{-1} \mathrm{del}$ primer corte que oscilo entre 0.8 y 1.2 (Tabla 2), se podría considerar a estos cultivares como alternativas doble propósito. Esta propuesta se debe a que los cultivares Klein Gladiador y Yarará fueron evaluados en la red de cultivares de la EEA INTA SDE (Estación Experimental Agropecuaria del Instituto Nacional de Tecnología Agropecuaria de Santiago del Estero, Argentina), obteniendo rendimientos medios en tn grano $\mathrm{ha}^{-1}$ de 2.7 y 2.4, respectivamente. En este sentido, López (2014), al evaluar líneas avanzadas y cultivares de trigo como doble propósito en la EEA INTA Bordenave en el periodo 2007-2012, determinó rendimientos de biomasa forrajera que oscilaron entre las 1.6 y 2 tn $\mathrm{ms} \mathrm{ha}^{-1}$, y rendimientos en grano que oscilaron entre las 1.7 y 2.6 tn grano ha-1.

Fueron determinadas fuertes relaciones directas e inversas entre coordenadas cromáticas y PBFA (tn ms $\mathrm{ha}^{-1}$ ) de variedades de trigo. Las mejores relaciones encontradas fueron entre la $\Sigma$ cct y la PBFA acumulada de las variedades Yarará, Serpiente y Titanio, mientras que con Gladiador se obtuvo la mejor relación con la PBFA del cuarto corte (Tabla 3). Similares resultados obtuvieron Pan et al. (2007) quienes determinaron que la mejor relación entre la producción de biomasa superficial de trigo y el porcentaje de cobertura derivado de imágenes de cámaras digitales se dio a los 80 días post siembra $(\mathrm{r}=0.60)$. A su vez, Kawashima \& Nakatani (1998) relacionaron la concentración de clorofila de trigo y centeno con funciones derivadas de las longitudes roja, verde y azul. Ellos encontraron

Tabla 3. Coeficiente de correlación de Pearson (r) entre coordenadas cromáticas integradas $(\Sigma)$ y la PBFA acumulada y por corte de cada variedad.

\begin{tabular}{lcccc}
\hline $\begin{array}{l}\text { Coordenadas } \\
\text { Cromáticas }\end{array}$ & $\begin{array}{c}\text { YARARA } \\
\text { Acumulada } \\
\text { tn } \mathrm{ms} \mathrm{ha}^{-1}\end{array}$ & $\begin{array}{c}\text { GLADIADOR } \\
\text { Corte } 4 \\
\text { tn } \mathrm{ms} \mathrm{ha}^{-1}\end{array}$ & $\begin{array}{c}\text { SERPIENTE } \\
\text { Acumulado } \\
\text { tn } \mathrm{ms} \mathrm{ha}^{-1}\end{array}$ & $\begin{array}{c}\text { TITANIO } \\
\text { Acumulado } \\
\text { tn } \mathrm{ms} \mathrm{ha}^{-1}\end{array}$ \\
\hline$\Sigma \Sigma$ cct & $\mathbf{- 0 . 9 9}$ & $\mathbf{- 0 . 9 9}$ & $\mathbf{0 . 9 6}$ & $\mathbf{- 0 . 8 8}$ \\
$\Sigma \Sigma$ ccr (red) & $\mathbf{- 0 . 9 9}$ & $\mathbf{- 0 . 9 9}$ & $\mathbf{0 . 9 2}$ & $\mathbf{- 0 . 8 3}$ \\
\hline $\begin{array}{l}\text { Todas las relaciones fueron altamente significativas }(\mathrm{p}<0.01) . \\
\text { seca por hectárea. }\end{array}$
\end{tabular}


Tabla 4. Modelos de regresión lineal simple $(\alpha$ 0.05) de PBFA por cultivar (tn ms ha ${ }^{-1}$ y coordenadas cromáticas

\begin{tabular}{llll}
\hline Variedades & $\mathbf{y}=\mathbf{m x}+\mathbf{b}$ & $\boldsymbol{p}$-valor & $\mathbf{R}^{\mathbf{2}}{ }_{\mathbf{A j}}$ \\
\hline SERPIENTE & PBFA $_{\text {acum }}=2.0 \Sigma \mathrm{cct}-11.4$ & $<0.01$ & 0.90 \\
TITANIO & PBFA $_{\text {acum }}=-7.74 \Sigma$ cctcct +74.54 & $<0.01$ & 0.71 \\
GLADIADOR & PBFA 4 corte $=-3.02 \Sigma$ cctcct $+28.57<0.01$ & 0.99 \\
YARARÁ & PBFA $_{\text {acum }}=-4.9 \sum$ cctcct +46.43 & $<0.01$ & 0.97 \\
\hline \multicolumn{2}{l}{ Medias con una letra común no son significativamente diferentes $(\mathrm{p}>0.05)}$. &
\end{tabular}

fuertes relaciones inversas entre el contenido de clorofila con los valores digitales diarios de $r-b$ y $g-b$ ( $\mathrm{r}$ $=-0.84$ a $r=-0.96)$, y al integrar los valores digitales de la estación de crecimiento la relación disminuyo $(\mathrm{r}=-$ 0.76). A su vez, los autores utilizaron la función $(r$ $b) /(r+b)$ para normalizar y reducir el ruido, encontrando las mejores relaciones con la concentración de clorofila con un $\mathrm{r}-0.81$, esta función resultó la más estable ante variaciones climáticas. Adamsen et al. (1999) cuantificaron la tasa de senescencia en trigo mediante la relación Green/Red derivada de cámara digital, y la relacionaron con el NDVI de espectrómetro manual y la concentración de clorofila en hoja derivada del SPAD 502 meter. Ellos determinaron fuertes $\mathrm{y}$ significativas relaciones directas entre la relación $G / R$ y el NDVI $\left(\mathrm{R}^{2}=0.96\right)$ y SPAD $\left(\mathrm{R}^{2}=0.87\right)$ concluyendo que las cámaras digitales tienen gran potencialidad para ser utilizadas como herramientas de monitoreo del estado de los cultivos.

Es importante destacar que mediante la simple coordenada cromática integrada $(\Sigma \mathrm{ccr})$ se obtuvieron fuertes relaciones directas e inversas con la PBFA. Resultados similares obtuvieron $\mathrm{Li}$ et al. (2010) mediante datos de reflectancia RGB derivados de cámaras digitales; ellos encontraron fuertes relaciones directas entre el porcentaje de cobertura de trigo con el contenido de nitrógeno $(\mathrm{r}=0.96)$, con la biomasa seca $(\mathrm{r}=0.97)$ y con el índice de área foliar $(\mathrm{r}=0.93)$, concluyendo que las bandas roja y verde indican correctamente el porcentaje de cobertura del follaje en estado vegetativo en invierno y comienzos de primavera. A su vez, Jía et al. (2009) relacionaron biomasa y contenido de nitrógeno total en plantas de trigo y maíz con números digitales $\mathrm{R}, \mathrm{G}, \mathrm{B}$ e índices $\mathrm{G} / \mathrm{R}, \mathrm{G} / \mathrm{B}, \mathrm{R} / \mathrm{B}, \mathrm{G} /(\mathrm{R}+\mathrm{G}+\mathrm{B}), \mathrm{R} /(\mathrm{R}+\mathrm{G}+\mathrm{B})$. Ellos encontraron fuertes relaciones inversas entre biomasa y contenido de nitrógeno, respectivamente con los índices $\mathrm{R} /(\mathrm{R}+\mathrm{G}+\mathrm{B})(r=-0.87 ; r=-0.95)$ y $\mathrm{G} /(\mathrm{R}+\mathrm{G}+\mathrm{B})$ $(r=-0.74 ; r=-0.84)$ y fuertes relaciones directas con el índice $\mathrm{B} /(\mathrm{R}+\mathrm{G}+\mathrm{B}) \quad(r=-0.83 ; r=0.92)$, $\mathrm{y}$ concluyeron que el mejor índice para determinar contenido de nitrógeno en trigo fue $\mathrm{R} /(\mathrm{R}+\mathrm{G}+\mathrm{B})$.

$\mathrm{La}$ relación directa encontrada entre las coordenadas cromáticas y la PBFA de la variedad Serpiente estaría relacionada con que esta variedad presenta diferencias en el área verde proyectada o bien una concentración de clorofila diferente con respecto al resto de las variedades. Esta respuesta fue comunicada por Tucker (1979) quien relacionó la fracción verde y roja del espectro electromagnético con la producción de biomasa en trigo, concluyendo que la fracción roja tiene fuerte relación no lineal inversa con la productividad de biomasa verde del trigo y que la fracción roja y verde son inversamente proporcionales a la vegetación fotosintéticamente activa en trigo. En este sentido, Ge et al. (2016) utilizaron el índice $2 \mathrm{G} /(\mathrm{B}+\mathrm{R})$ para determinar parámetros biofísicos en hojas de maíz (Zea mays). Ellos determinaron fuertes relaciones directas entre el índice y el área verde proyectada $(r=0.98)$, materia verde $(r=0.99)$ y con materia seca $(r=0.95)$, concluyendo que el área proyectada derivada del índice y relacionada con mediciones destructivas es un buen estimador de la materia verde, materia seca y área de hoja en estados vegetativos tempranos $(r=0.95)$. Los modelos lineales ajustados entre la PBFA de las variedades de trigo y las coordenadas cromáticas presentaron un elevado poder explicativo, los coeficientes de determinación ajustados de los modelos oscilaron entre 0.71 y 0.99 .

\section{Conclusiones.}

1. Las variedades de trigo analizadas tuvieron un buen comportamiento en cuanto a productividad de biomasa forrajera aérea en el área de estudio.

2. Las coordenadas cromáticas integradas, totales y simples, se relacionan, directa o indirectamente, con la productividad de biomasa forrajera aérea de variedades de trigo. Posibilitando, de manera simple y económica, cuantificarla.

3. Los modelos lineales ajustados toman relevancia por su potencial utilidad para predecir la productividad de biomasa forrajera aérea de las variedades de trigo.

\section{Agradecimientos.}

- Al Criadero Klein S.A (www.trigoklein.com.ar) por el material genético aportado para la realización de los ensayos en el campo experimental de la FCN Sede Sur UNSa, Metan, Salta y en establecimiento JB en Col. El Simbolar, Santiago del Estero.

- Al Prof. Ing. Ramón Ledesma - Cat. Ingl\{es Técnico - FCF - UNSE por el Summary.

- El establecimiento JB, es uno de los seis establecimientos de la agricultura familiar de Santiago del Estero que forman parte del proyecto CyT UNSE denominado - Productividad primaria 
neta aérea de los sistemas pastoriles de alfalfa (Medicago sativa $\mathrm{L}$ ) de predios de la agricultura familiar de Los Pereyra, Dpto. Robles y su relación con Índices de Vegetación derivados de sensores remotos y cercanos. Director Dr. Ing. Tiedemann JL (actualmente en evaluación).

\section{Literatura citada.}

Aase J. K. \& Siddoway F. H. 1981. Assessing Winter Wheat Dry Matter Production Via Spectral Reflectance Measurements. Remote Sensing of Environment. 11:267-277.

Adamsen F. J. P. J., Pinter P. J., Barnes E. M., LaMorte R. L., Wall G. W., Leavitt S. W. \& Kimball B. A. 1999. Measuring wheat senescence using a digital camera. Crop Sci. 39:719-724.

Ahrends H. E., Brugger R., Stockli R., Schenk J., Michna P., Jeanneret F., Wanner H.\& Eugster W. 2008. Quantitative phenological observations of a mixed beech forest in northern Switzerland with digital photography. J. Geophys. Res. 113, doi:10.1029/2007JG000650.

Ahrends H. E., Etzold S., Kutsch W. L., Stoeckli R., Bruegger R., Jeanneret F., Wanner H., Buchmann N. \& Eugster W. 2009. Tree phenology and carbon dioxide fluxes: use of digital photography for process-based interpretation at the ecosystem scale. Climate Research. 39:261-274.

Angueira C., Prieto D., Lopez J. \& Barraza G. 2007. Geomorfología. En: Sistemas de Información Geográfica de Santiago del Estero, CD ROOM, ISBN - 987-521170-2. Instituto de Tecnología Agropecuaria, EEA Santiago del Estero, Argentina.

Asrar G., Funchs M., Kanemasu E. T. \& Hatfield J. L. 1984 Estimating absorbed photosynthetic radiation and leaf area index from spectral reflectance in wheat. Agronomy Journal. 76:300-306.

Balzarini M. G., Gonzalez L., Tablada M., Casanoves F., Di Rienzo J. A. \& Robledo C. W. 2008. Manual del Usuario, Editorial Brujas, Córdoba, Argentina.

Baret F., de Solan B., Lopez-Lozano R., Ma K. \& Weiss M. 2010. GAI estimates of row crops from downward looking digital photos taken perpendicular to rows at $57.5^{\circ}$ zenith angle: Theoretical considerations based on $3 \mathrm{D}$ architecture models and application to wheat crops. Agricultural and Forest Meteorology. 150:1393-1401.

Boletta P. E. 2001. Utilización de información agrometereológica y satelital para la evaluación de la desertificación en el Chaco seco - Dpto Moreno, Santiago del Estero. Tesis para optar al grado de magíster en ciencias Agropecuarias, Dpto. de postgrado, Universidad Nacional de Córdoba.

Crespo J. R., Castaño J. A. \& Capurro J. A. 2007. Agricultura Técnica (Chile). 67:210-218.

Di Rienzo J. A., Casanoves F., Balzarini M. G., Gonzalez L., Tablada M. \& Robledo C. W. 2008. InfoStat, versión 2008, Grupo InfoStat, FCA, Universidad Nacional de Córdoba, Argentina.

Dye D. G. \& Goward S. N. 1993. Photosynthetically active radiation absorbed by global land vegetation in August 1984. Int. J. Remote Sensing. 14:3361-3364.

Fensholt R. 2003. Assessment of Primary Production in Semi-arid Environment from Satellite Data. Exploiting capabilities of new sensors. Ph. D. dissertation, Institute of Geography, University of Copenhagen, Copenhagen $\mathrm{K}$, Denmark, 87p.

Fensholt R., Sandholt I., Rasmussen M., Stisen S. \& Diouf A. 2006. Evaluation of satellite based primary production modelling in the semiarid Sahel, Remote Sensing of Environment. 105:173-188.

Ferreira T. \& Rasband W. 2016. ImageJ User Guide. Disponible en: http://imagej.nih.gov/ij/docs/guide.

Field C. B., Randerson J. T. \& Malmstriik C. M. 1995. Global Net Primary Production: Combining Ecology and Remote Sensing. Remote Sensing Environment. 51: 7488.

Gardner A. L. 1974. Producción y utilización de pasturas. En: curso de producción animal para extensionistas, INTA Balcarce. Ed. GARDNER A. L. Proyecto FAO ARG 527, EERA INTA Balcarce, 162pp.

Ge Y., Bai G., Stoerger V. \& Schnable J. C. 2016. Temporal dynamics of maize plant growth, water use, and leaf water content using automated high throughput RGB and hyperspectral imaging. Computers and Electronics in Agriculture. 127:625-632.

Gillespie A. R., Kahle A. B., Kahle A. B. \& Walker R. E. 1986. Color enhancement of highly correlated images. I. Decorrelation and HSI Contrast Stretches. Remote Sens. Environ. 20:209-235.

Gillespie A. R., Kahle A. B. \& Walker R. E. 1987. Color enhancement of highly correlated images. II. Channel ratio and chromaticity transformation techniques. Remote Sens. Environ. 22:343-365.

Gitelson A. A., Kaufman Y. J., Stark R. \& Rundquist D. 2002. Novel Algorithms for Remote Estimation of Vegetation Fraction, Remote Sensing of Environment. 80:76- 87

Goetz S. J., Prince S. D., Goward S. N., Thawley M. M., Small J. \& Johnston A. 1999. Mapping net primary production and related biophysical variables with remote sensing: Application to the BOREAS region. J. Geophys. Res., 104(D22), 7719-27734, doi:10.1029/1999JD900269.

Ide R. \& Oguma H. 2010. Use of digital cameras for phenological observations. Ecological Informatics. 5: 339-347.

Inoue T., Nagai S., Kobayashi H. \& Koizumi H. 2015. Utilization of ground-based digital photography for the evaluation of seasonal changes in the aboveground green biomass and foliage phenology in a grassland ecosystem. Ecological Informatics. 25:1-9

Jia L., Chen X., Li M., Cui Z. \& Zhang F. 2009. Comparsion of multispectral reflectance with digital color image in assessing the winter wheat nitrogen status. Disponible en: https://link.springer.com/content/pdf/10.1007/978-14419-0211-5_43.pdf

Kawashima S. \& Nakatani M. 1998. An algorithm for estimating growth and development on the pathway of floral development, chlorophyll content in leaves using a video camera. Annals of Botany 81:49-54.

Lee K. \& Lee B. 2013. Estimation of rice growth and nitrogen nutrition status using color digital camera image analysis. Europ. J. Agronomy. 48:57-65

Li Y., Chena D., Walker C. N. \& Angus J. F. 2010. Estimating the nitrogen status of crops using a digital camera. Field crops Research 118:221-227.

López J. 2014. Estrategias de mejoramiento y evaluación del comportamiento doble propósito (pasto y grano) de trigo y triticale en la EEA INTA Bordenave. En: Verdeos de 
invierno. Editor Marcelo Ortellado. $1^{\text {a }}$ ed. Bordenave, Buenos Aires, Ediciones INTA, p52.

Lukina E. V., Stone M. L. \& Raun W.R. 1999. Estimating vegetation Coverage in Wheat Using Digital Image. Journal of Plant Nutrition 22:341-350.

Mizunuma T., Koyanagi T., Mencuccini M., Nasahara K. N., Wingate L. \& Grace J. 2011. The comparison of several colour indices for the photographic recording of canopy phenology of Fagus crenata Blume in eastern Japan, Plant Ecology \& Diversity. Disponible en: https://doi.org/10.1080/17550874.2011.563759.

Nouvellon Y., Seen D. L., Rambal S., Begé A., Moran M., Kerr Y. \& Qi J. 2000. Time Course of Radiation Use Efficiency in a Shortgrass Ecosystem: Consequences for Remotely Sensed Estimation of Primary Production. Remote Sens. Environ. 71:43-55.

Pan G., Li F. M. \& Sun G. J. 2007. Digital camera based measurement of crop cover for wheat yield prediction. Proceedings of IGARSS 2007: IEEE International Geoscience and Remote Sensing Symposium, Barcelona, Spain, 23-28 July 2007; pp. 797-800.

Rasmussen J., Nørremark M. \& Bibby B. M. 2007. Assessment of leaf cover and crop soil cover in weed harrowing research using digital images. Weed Research, 47:299-310.

Sakamoto T., Shibayama M., Kimura A. \& Takada E. 2011. Assessment of digital camera-derived vegetation indices in quantitative monitoring of seasonal rice growth. ISPRS Journal of Photogrammetry and Remote Sensing 66:872-882.

Sakamoto T., Gitelson A. A., Nguy-Robertson A. L., Arkebauer J., Wardlowb B. D., Suyker A. E., Vermab S. B. \& Shibayama M. 2012. An alternative method using digital cameras for continuous monitoring of crop status. Agricultural and Forest Meteorology 154-155:113-126.

Tiedemann J. L. 2011a. Fenología del bosque de las subregiones naturales: Chaco semiárido y Chaco serrano, de Santiago del Estero, Argentina. Ecol. apl.10:50-58

Tiedemann J.L. 2011b. Dinámica espacial y temporal del Índice de Vegetación de Diferencia Normalizada en Santiago del Estero. Tesis Doctoral, Escuela de Graduados Universidad Nacional de Córdoba, Argentina, $157 \mathrm{p}$.

Tiedemann J.L. 2015. Fenología y productividad primaria neta aérea de sistemas pastoriles de Panicum maximum en el Dpto. Moreno, Santiago del Estero, Argentina, derivada del NDVI MODIS. Ecol. apl. 14: 27-39.

Torres Bruchmann E. 1981. Climatología General y Agrícola de la Provincia de Santiago del Estero. Universidad Nacional de Tucumán, Tucumán, Argentina, 199 pp.

Tucker C. J. 1979. Red and photographic infrared linear combinations for monitoring vegetation Remote Sens Environ 8.127-150.

Tucker C. J. \& Sellers P. J. 1986. Satellite remote sensing of primary production. Int. J. Remote Sensing 11:13951416.

Wiegand C. L., Richardson A. J. \& Kanemasu E. T. 1979. Leaf area index estimates for wheat from LANDSAT and their Implications for evapotranspiration and crop modeling. Agron j. 71:336-342.

Woebbecke D. M., Meyer G. E., Von Bargen K. \& Mortensen D. A. 1995. Color indices for weed identification under various soil, residue, and lighting conditions. Transactions of the ASAE. 38:259-269.

Yen J., Chang F. \& Chang S.1995. A new criterion for automatic multilevel thresholding. IEEE Transactions. 4:370-378.

\footnotetext{
${ }^{1}$ Cátedra de Forrajes - Sede regional Sur, Facultad de Ciencias Naturales, Universidad Nacional de Salta; Becario Postdoctoral MINCyT-UNSE.
} 